\title{
Diffusion of Chemically Reactive Species in Casson Fluid Flow over an Unsteady Stretching Surface in Porous Medium in the Presence of a Magnetic Field
}

\author{
Gilbert Makanda, Sachin Shaw, and Precious Sibanda \\ School of Mathematics, Statistics and Computer Science, University of KwaZulu-Natal, Private Bag X01, Scottsville, \\ Pietermaritzburg 3209, South Africa
}

Correspondence should be addressed to Precious Sibanda; sibandap@ukzn.ac.za

Received 31 July 2014; Accepted 14 September 2014

Academic Editor: Stanford Shateyi

Copyright (C) 2015 Gilbert Makanda et al. This is an open access article distributed under the Creative Commons Attribution License, which permits unrestricted use, distribution, and reproduction in any medium, provided the original work is properly cited.

A study is performed on two-dimensional flow and diffusion of chemically reactive species of Casson fluid from an unsteady stretching surface in porous medium in the presence of a magnetic field. The boundary layer velocity, temperature, and concentration profiles are numerically computed for different governing parameters. The paper intends to show unique results of a combination of heat transfer and chemical reaction in Casson fluid flow. The resulting partial differential equations are converted to a system of ordinary differential equations using the appropriate similarity transformation, which are solved by using the RungeKutta-Fehlberg numerical scheme. The results in this work are validated by the comparison with other authors.

\section{Introduction}

The study of Casson fluid has attracted attention to many researchers due to its application in the field of metallurgy, food processing, drilling operations, and bioengineering operations. Its application extends to the manufacturing of pharmaceutical products, coal in water, china clay, paints, synthetic lubricants, and biological fluids such as synovial fluids, sewage sludge, jelly, tomato sauce, honey, soup, and blood due to its contents such as plasma, fibrinogen, and protein, making the study of Casson fluid important in fluid dynamics. Casson fluid is classified as a non-Newtonian fluid due to its rheological characteristics. These characteristics show shear stress-strain relationships that are significantly different from Newtonian fluids. The study of nonNewtonian fluids has not been thoroughly covered due to the complex representation of their constitutive equations. It is therefore important to undertake this study of Casson fluid. Most studies have concentrated on viscoelastic fluids in which different constitutive equations have been suggested. This work can be applied to chemical processing equipment in which some fluids react chemically with some ingredients present in them.
The driving force for mass transfer is a combination of temperature and concentration gradients. In this study the effect of chemical reaction on the fluid is considered as in Mukhopadhyay and Vajravelu [1]. The study of boundary layer flow over a stretching sheet has been studied by Mukhopadhyay et al. [2], among others, who investigated Casson fluid flow over an unsteady stretching surface. In their work they did not consider mass transfer and they considered a different wall temperature expression. Abd El-Aziz [3] studied mixed convection flow of a micropolar fluid from an unsteady stretching surface with viscous dissipation. In this work he considered a similar stretching velocity, wall temperature, and wall concentration distribution. We extended the work of Grubka and Bobba [4] who investigated heat transfer characteristics of a continuous stretching surface with variable temperature in which we introduced the MHD and porous medium source terms and chemical reaction effects. Sharidan et al. [5] studied similarity solutions for the unsteady boundary layer flow and heat transfer due to a stretching sheet; Nadeem et al. [6,7] and Ahmed and Nazar [8] also studied Casson fluid over a stretching sheet and in their paper they assumed that the velocity of the stretching 
surface is linearly proportional to the distance from fixed origin.

In the study of non-Newtonian fluids many authors have studied the flow of blood as Casson fluid. The studies were carried out by Rohlf and Tenti [9], among others, who investigated the role of Womersley number in pulsatile blood flow, a theoretical study of the Casson model; Sankar and Lee $[10,11]$ investigated two-fluid nonlinear model for flow in catheterized blood vessels and two-fluid Casson model for pulsatile blood flow through stenosed arteries, respectively. Shaw et al. [12] studied pulsatile Casson fluid flow through stenosed bifurcated artery. In relation to blood flow there are other research works that were done in different geometries such as flows in microslit channels, slightly curved channels, and peristaltic transport as in [13-15].

The study of Casson fluid in porous media was also studied by Nadeem et al. [16] who considered MHD threedimensional Casson fluid flow past a porous linearly stretching sheet. Dash et al. [17] studied Casson fluid flow in a pipe filled with homogeneous porous medium. Tripathi [18] investigated the transient peristaltic heat flow through a finite porous channel. Pramanik [19] studied Casson fluid flow and heat transfer past an exponentially porous stretching surface in the presence of thermal radiation. Ramachandra et al. [20] investigated flow and heat transfer of Casson fluid from a horizontal circular cylinder with partial slip in a nonDarcy porous medium. In their work they considered slip conditions on the wall.

There are many studies that investigated fluid flow with chemical reactions. Kameswaran et al. [21] investigated homogeneous-heterogeneous reactions in a nanofluid flow due to a porous stretching sheet, Shaw et al. [22] studied homogeneous-heterogeneous reactions in a nanofluid flow due to a porous stretching sheet, and Chamkha et al. [23] investigated similarity solutions for unsteady heat and mass transfer from a stretching surface embedded in a porous medium with suction/injection and chemical reaction effects.

Although there are many applications and use of nonNewtonian fluids in industry, the study of Casson fluid has not been thoroughly investigated for heat and mass transfer past a stretching surface. In this work we extend the work of Mukhopadhyay and Vajravelu [1] and Grubka and Bobba [4] in which the energy equation, the source terms for porous medium, and magnetic field are introduced. Similarity transformations are used to convert the partial differential equations into ordinary differential equations which are then solved by using Runge-Kutta-Fehlberg integration scheme and the successive linearization method described by Makanda et al. [24]. In this work we investigate the effect of varying unsteadiness parameter, Casson, Schmidt, and Prandtl numbers, and the reaction rate parameter on the velocity, temperature, and concentration profiles with the depiction of graphical illustrations.

\section{Mathematical Formulation}

Consider two-dimensional laminar boundary layer flow, temperature, and mass transfer of an incompressible Casson fluid flow over an unsteady stretching sheet. The flow of heat and mass transfer starts at $t=0$. The sheet is pulled out of the slit at the origin $(x=0, y=0)$ and moves with velocity $U(x, t)=a x /(1-\alpha t), a>0, \alpha \geq 0$ are constants, and $a$ is the initial stretching rate. The rheological equation of state for an isotropic and incompressible flow of a Casson fluid is given as in $[1,2,20]$ by

$$
\tau_{i j}= \begin{cases}2\left(\mu_{B}+\frac{P_{y}}{\sqrt{2 \pi}}\right) e_{i j}, & \pi>\pi_{c} \\ 2\left(\mu_{B}+\frac{P_{y}}{\sqrt{2 \pi_{c}}}\right) e_{i j}, & \pi<\pi_{c},\end{cases}
$$

where $\pi=e_{i j} e_{i j}$ and $e_{i j}$ is the $(i, j)$ th component of the deformation rate, $\pi$ is the product of the deformation rate with itself, $\pi_{c}$ is a critical value of this product based on the non-Newtonian model, $\mu_{B}$ is the plastic dynamic viscosity of the non-Newtonian fluid, and $P_{y}$ is the yield stress of the fluid. Given that $T_{w}$ and $C_{w}$ are, respectively, the temperature and concentration at the sheet and $T_{\infty}$ and $C_{\infty}$ are, respectively, the ambient conditions, the positive $x$ coordinate is measured along the stretching sheet and the positive $y$ coordinate is measured perpendicular to the sheet. It is assumed that both temperature and concentration at the surface vary with distance from the origin and time. The temperature $T_{w}$ and concentration $C_{w}$ at the surface are therefore given by

$$
T_{w}(x, t)=T_{\infty}+\frac{b x}{(1-\alpha t)^{2}}, \quad C_{w}(x, t)=C_{\infty}+\frac{c x}{(1-\alpha t)^{2}},
$$

where $b$ and $c$ are constants. The surface temperature and surface concentration increase if $b$ and $c$ are positive and reduce if they are negative from $T_{\infty}$ and $C_{\infty}$ at the origin to $x$ and the temperature and concentration increase/decrease along the sheet. It is assumed that radiation effects and viscous dissipation are negligible. The expressions $U_{w}(x, t)$, $T_{w}(x, t)$, and $C_{w}(x, t)$ are only valid for $t<\alpha^{-1}$ but not when $\alpha=0$. Under these assumptions the governing equations in this flow are given as

$$
\begin{gathered}
\frac{\partial}{\partial x}(u)+\frac{\partial}{\partial y}(v)=0 \\
\frac{\partial u}{\partial t}+u \frac{\partial u}{\partial x}+v \frac{\partial u}{\partial y}=v\left(1+\frac{1}{\beta}\right) \frac{\partial^{2} u}{\partial y^{2}}-\frac{v}{K} u-\frac{\sigma B_{0}^{2}}{\rho} u \\
\frac{\partial T}{\partial t}+u \frac{\partial T}{\partial x}+v \frac{\partial T}{\partial y}=\alpha_{0} \frac{\partial^{2} T}{\partial y^{2}} \\
\frac{\partial C}{\partial t}+u \frac{\partial C}{\partial x}+v \frac{\partial C}{\partial y}=D \frac{\partial^{2} C}{\partial y^{2}}-k\left(C-C_{\infty}\right),
\end{gathered}
$$

where $\gamma$ is kinematic viscosity of Casson fluid, $\beta=$ $\mu_{B} \sqrt{2 \pi_{c}} / P_{y}$ is the non-Newtonian Casson parameter, $K$ is the permeability of the porous medium, $\sigma$ is the electrical conductivity, $B_{0}$ is the strength of the magnetic field, $\rho$ is the density of the Casson fluid, $D$ is the diffusion coefficient of species in the fluid, $\alpha_{0}$ is the thermal diffusivity, and $k(t)=$ $k_{0} /(1-\alpha t)$ is the time dependent reaction rate, where $k>0$ 


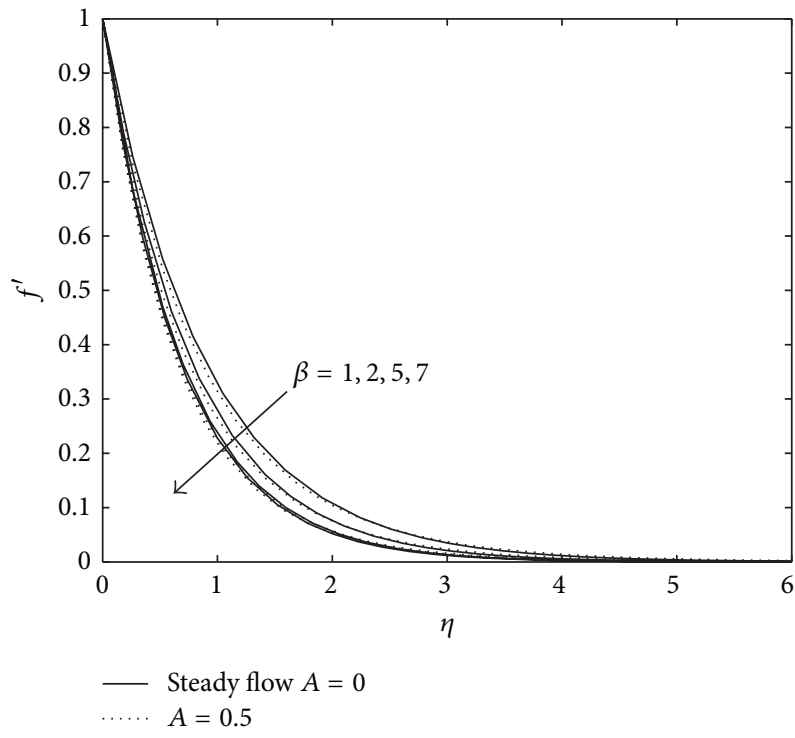

(a) Velocity profiles for different values of the Casson parameter $\beta$ and unsteadiness parameter $A$ at $\Lambda=0.5, M=1, \operatorname{Pr}=1, \mathrm{Sc}=1$, and $R=0.5$

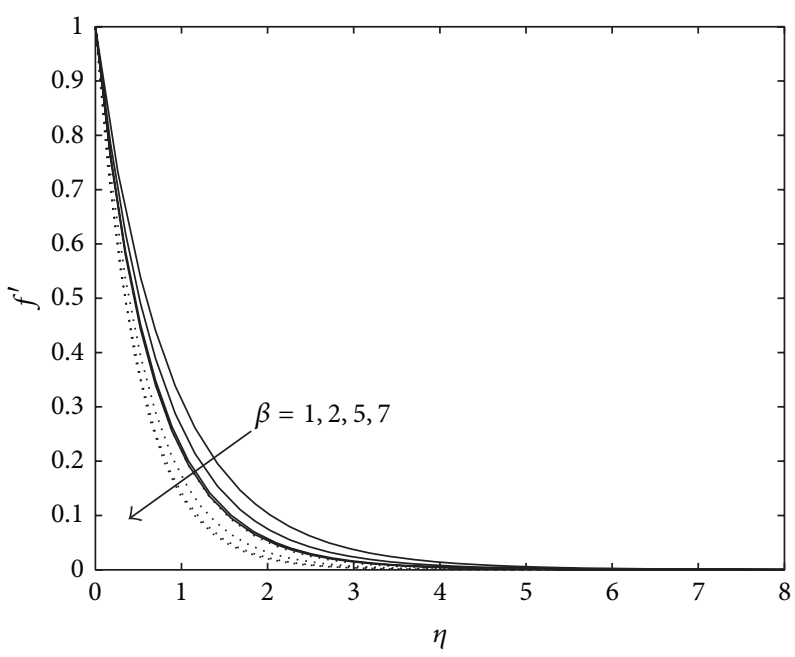

$$
-M=1
$$$$
M=3
$$

(c) Velocity profiles for different values of the Casson parameter $\beta$ and magnetic parameter $M$ at $A=0.5, \Lambda=0.5, \operatorname{Pr}=1, \mathrm{Sc}=1$, and $R=0.5$

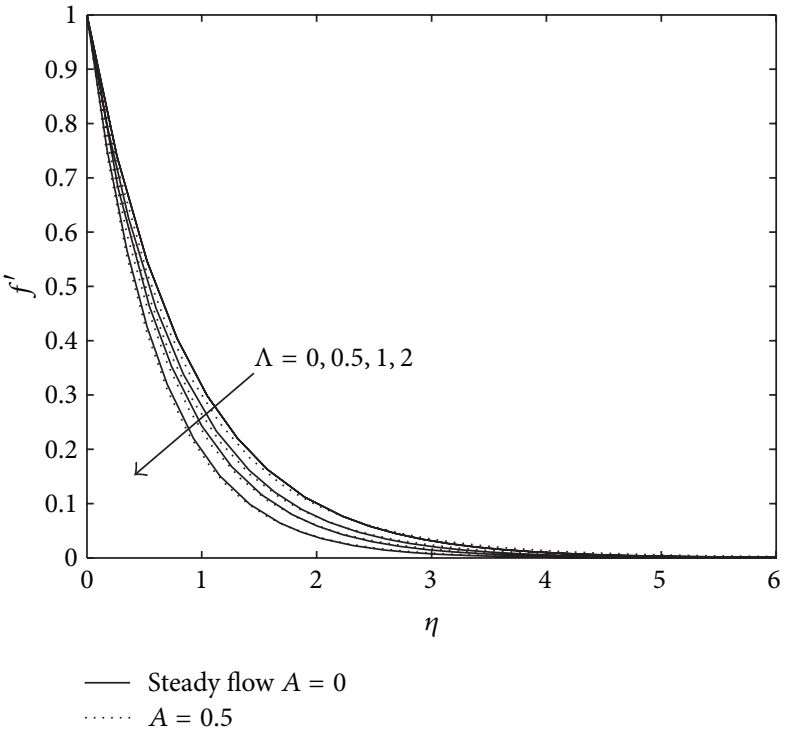

(b) Velocity profiles for different values of the permeability parameter $\Lambda$ and unsteadiness parameter $A$ at $\beta=2, M=1, \operatorname{Pr}=1, \mathrm{Sc}=1$, and $R=0.5$

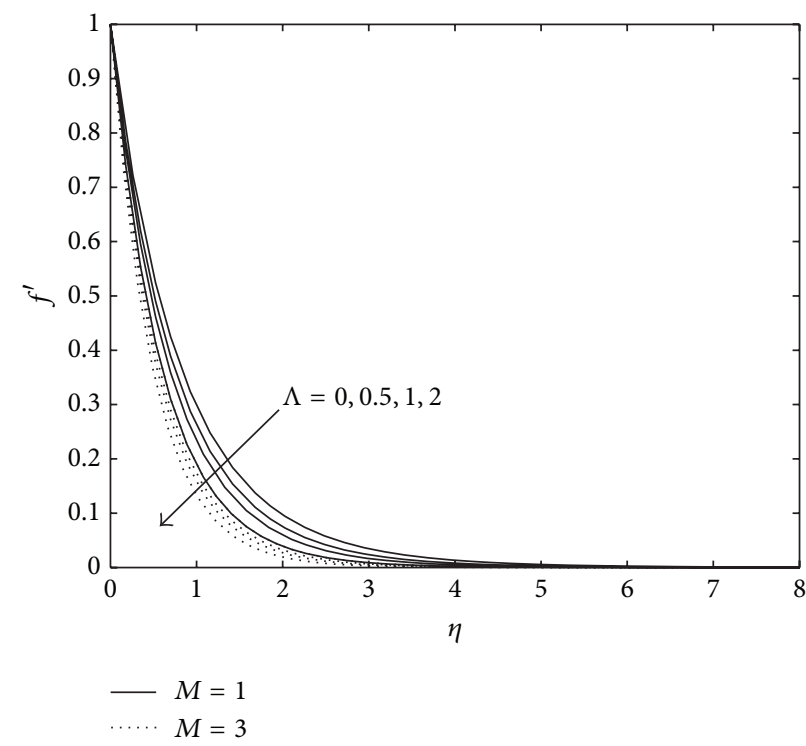

(d) Velocity profiles for different values of the permeability parameter $\Lambda$ and magnetic parameter $M$ at $\beta=2, A=0.5, \operatorname{Pr}=1, \mathrm{Sc}=1$, and $R=0.5$

FIGURE 1: Effects of different parameters on velocity profiles.

represents destructive reaction, $k<0$ represents constructive reaction, and $k_{0}$ is a constant. The boundary conditions are given as

$$
\begin{aligned}
& u=U(x, t), \quad v=0, \quad T=T_{w}(x, t), \\
& C=C_{w}(x, t), \quad y=0, \\
& u \longrightarrow 0, \quad T \longrightarrow T_{\infty}, \quad C \longrightarrow C_{\infty}, \quad \text { as } y \longrightarrow \infty,
\end{aligned}
$$

where the subscript $\infty$ refers to the ambient condition.
We introduce the nondimensional variables

$$
\begin{gathered}
u=\frac{\partial \psi}{\partial y}, \quad v=-\frac{\partial \psi}{\partial x} \\
\eta=\sqrt{\frac{a}{v(1-\alpha t)}} y, \quad \psi=\sqrt{\frac{\nu a}{1-\alpha t}} x f(\eta), \\
T_{w}(x, t)=T_{\infty}+\frac{b x}{(1-\alpha t)^{2}} \theta(\eta) \\
C_{w}(x, t)=C_{\infty}+\frac{c x}{(1-\alpha t)^{2}} \phi(\eta)
\end{gathered}
$$




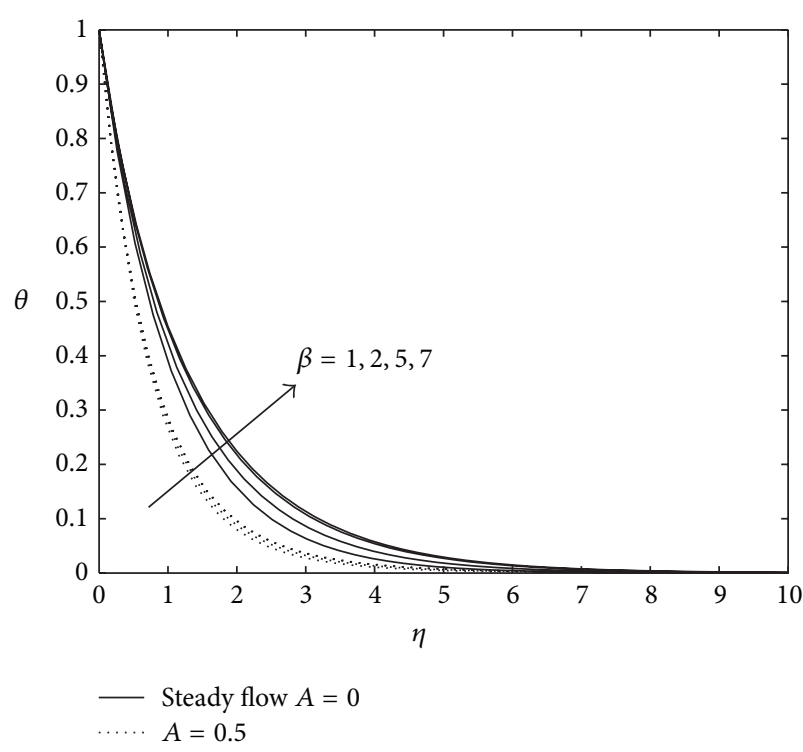

(a) Temperature profiles for different values of the Casson parameter $\beta$ and unsteadiness parameter $A$ at $\Lambda=0.5, M=1, \operatorname{Pr}=1, \mathrm{Sc}=1$, and $R=0.5$

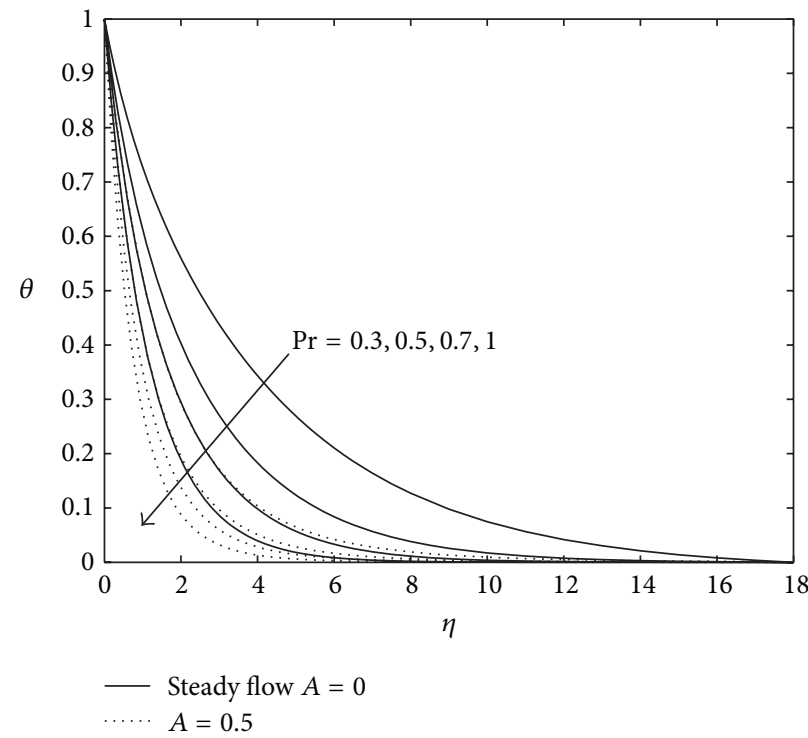

(c) Temperature profiles for different values of the Prandtl numbers Pr and unsteadiness parameter $A$ at $\beta=2, \Lambda=0.5, M=1, \mathrm{Sc}=1$, and $R=0.5$

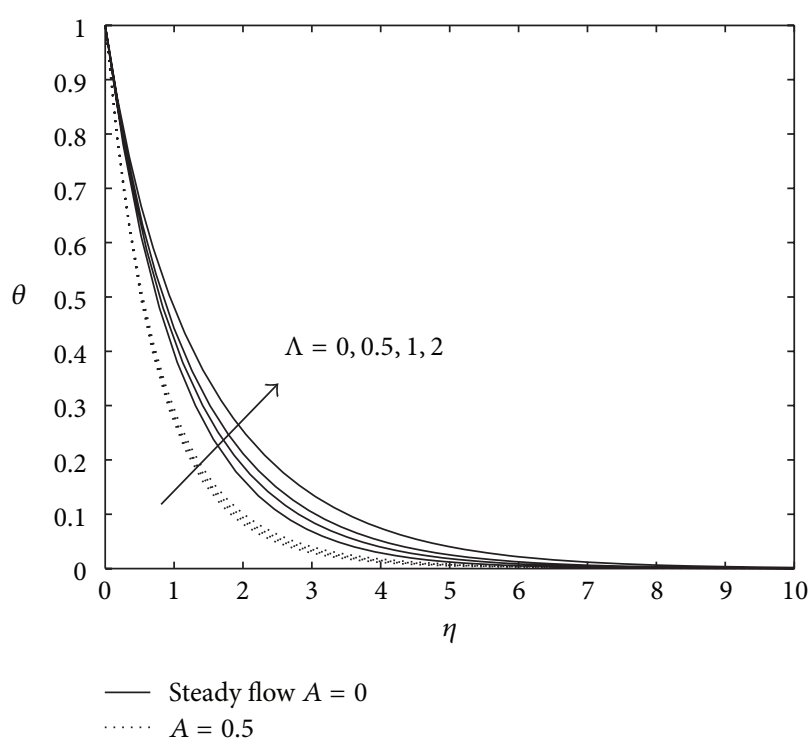

(b) Temperature profiles for different values of the permeability parameter $\Lambda$ and unsteadiness parameter $A$ at $\beta=2, M=1, \operatorname{Pr}=1, \mathrm{Sc}=1$, and $R=0.5$

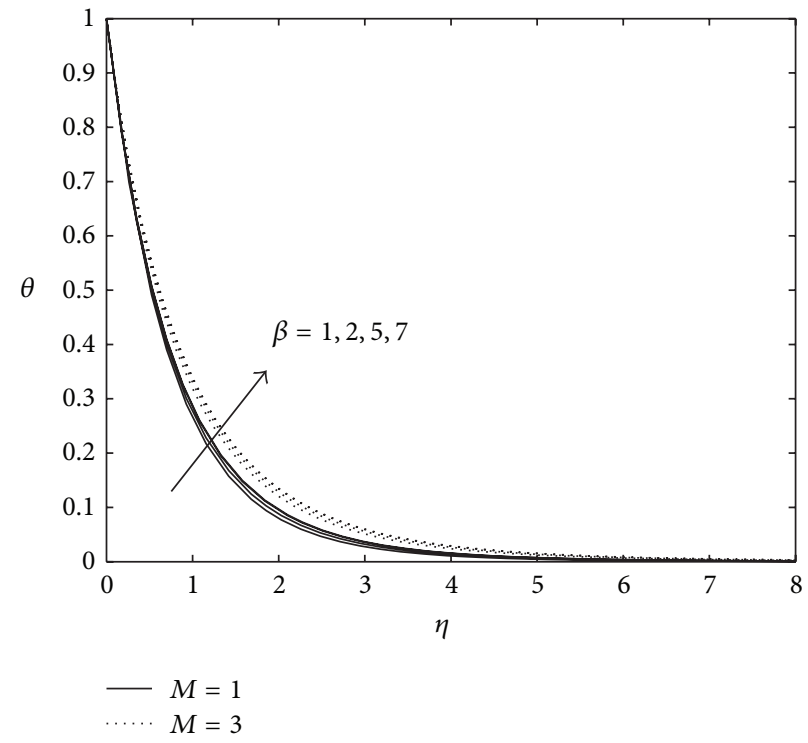

(d) Temperature profiles for different values of the Casson parameter $\beta$ and magnetic parameter $M$ at $A=0.5, \Lambda=0.5, \operatorname{Pr}=1, \mathrm{Sc}=1$, and $R=0.5$

FIGURE 2: Effects of different parameters on temperature profiles.

where $\psi(x, y, t)$ is the stream function which satisfies the continuity equation (3). The velocity components are defined as

$$
u=\frac{\partial \psi}{\partial y}=U_{w} f^{\prime}(\eta), \quad v=-\frac{\partial \psi}{\partial x}=-\sqrt{\frac{\nu a}{1-\alpha t}} .
$$

The governing equations reduce to

$$
\begin{gathered}
\left(1+\frac{1}{\beta}\right) f^{\prime \prime \prime}+f f^{\prime \prime}-f^{\prime 2}-\frac{A}{2}\left(2 f^{\prime}+\eta f^{\prime \prime}\right) \\
-\left(\Lambda+M^{2}\right) f^{\prime}=0,
\end{gathered}
$$

$$
\begin{gathered}
\frac{1}{\operatorname{Pr}} \theta^{\prime \prime}+f \theta^{\prime}-f^{\prime} \theta-\frac{A}{2}\left(4 \theta+\eta \theta^{\prime}\right)=0, \\
\frac{1}{\mathrm{Sc}} \phi^{\prime \prime}+f \phi^{\prime}-f^{\prime} \phi-\frac{A}{2}\left(4 \phi+\eta \phi^{\prime}\right)-R \phi=0,
\end{gathered}
$$

with boundary conditions

$$
\begin{aligned}
& f(0)=0, \quad f^{\prime}(0)=1, \quad \theta(0)=1, \quad \phi(0)=1, \\
& f^{\prime}(\infty) \longrightarrow 0, \quad \theta(\infty) \longrightarrow 0, \quad \phi(\infty) \longrightarrow 0,
\end{aligned}
$$




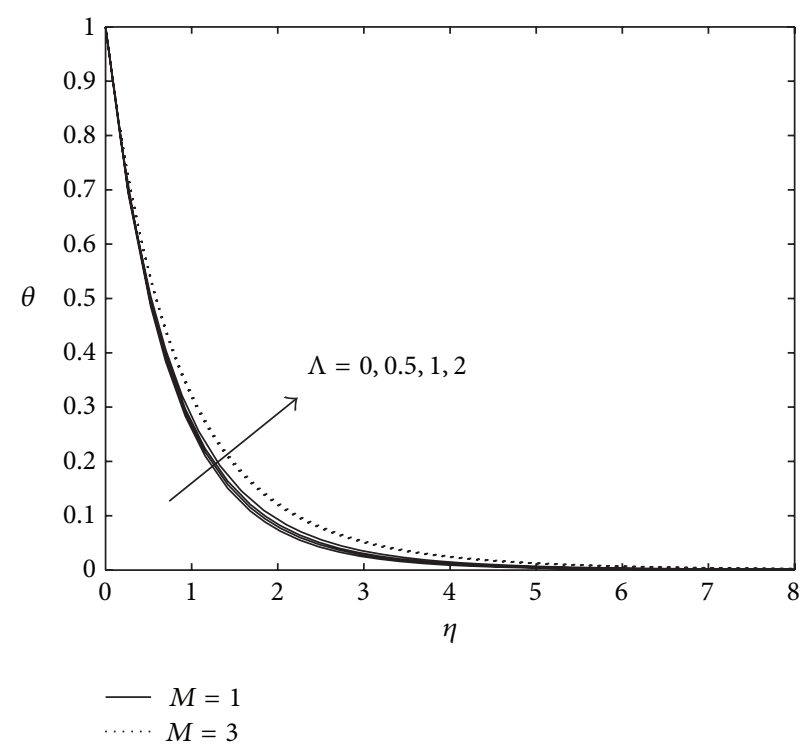

(a) Temperature profiles for different values of the permeability parameter $\Lambda$ and magnetic parameter $M$ at $\beta=2, A=0.5, \operatorname{Pr}=1, \mathrm{Sc}=1$, and $R=0.5$

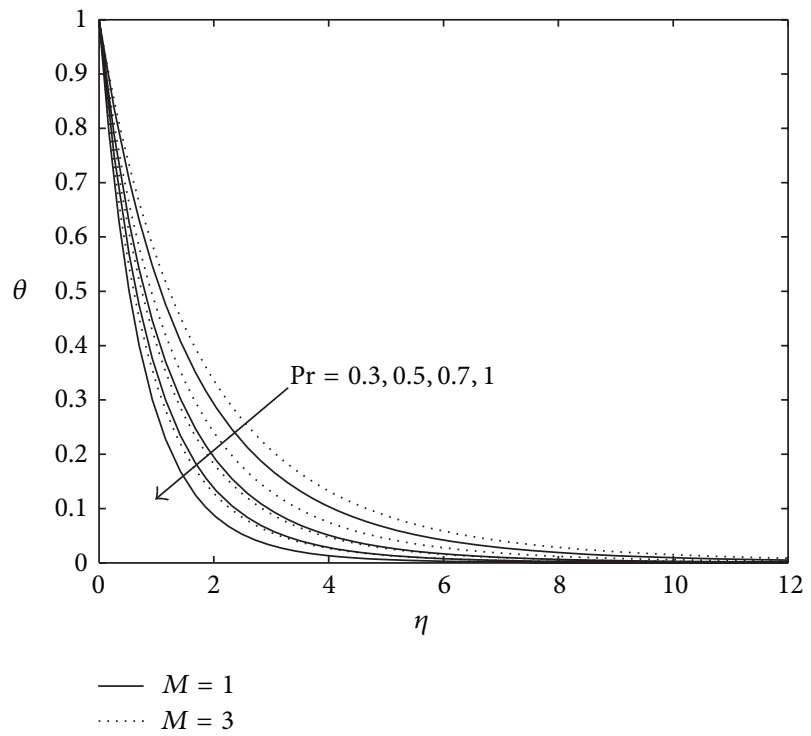

(b) Temperature profiles for different values of the Prandtl number $\operatorname{Pr}$ and magnetic parameter $M$ at $\beta=2, A=0.5, \Lambda=0.5, \mathrm{Sc}=1$, and $R=0.5$

FIGURE 3: Effects of different parameters on temperature profiles.

where $A=\alpha / a$ is the unsteadiness parameter, $\operatorname{Pr}=\nu / \alpha_{0}$ is the Prandtl number, Sc $=v / D$ is the Schmidt number, and $R=k_{0} / a$ is the reaction parameter. $\Lambda=v(1-\alpha t) / a K$ is the permeability parameter; $M^{2}=\sigma B_{0}^{2}(1-\alpha t) / \rho a$ is the magnetic parameter. The nondimensional temperature and concentration are, respectively, given by $\theta=\left(T-T_{\infty}\right) /\left(T_{w}-\right.$ $\left.T_{\infty}\right)$ and $\phi=\left(C-C_{\infty}\right) /\left(C_{w}-C_{\infty}\right)$.

The parameters of engineering interests are the local skin friction and the Nusselt and Sherwood numbers which are defined as

$$
C_{f x}=2\left(1+\frac{1}{\beta}\right) \operatorname{Re}_{x}^{-1 / 2} f^{\prime \prime}(0)
$$

The local Nusselt and Sherwood numbers are defined as

$$
\begin{aligned}
\mathrm{Nu}_{x}=\frac{x}{\alpha_{0}} \frac{q_{w}}{\left(T_{w}-T_{\infty}\right)}, & \mathrm{Sh}_{x}=\frac{x}{D} \frac{J_{w}}{\left(C_{w}-C_{\infty}\right)}, \\
q_{w}=-\alpha_{0}\left[\frac{\partial T}{\partial y}\right]_{y=0}, & J_{w}=-D\left[\frac{\partial C}{\partial y}\right]_{y=0} .
\end{aligned}
$$

Using expressions (11) and (6),

$$
\mathrm{Nu}_{x}=-\mathrm{Re}_{x}^{1 / 2} \theta^{\prime}(0), \quad \mathrm{Sh}_{x}=-\mathrm{Re}_{x}^{1 / 2} \phi^{\prime}(0),
$$

where $\mathrm{Re}_{x}$ is the Reynolds number defined as $\operatorname{Re}_{x}=U_{w} x / \nu$. It is important at this stage to mention that the system of equations (8)-(9) reduce to those of Grubka and Bobba [4] when $(1 / \beta \rightarrow 0), A=\lambda=M=\mathrm{Sc}=0$. The present problem reduces to that of Grubka and Bobba [4], $A=0$ denote steady flow, and in their paper they obtained an exact solution in terms of Kummer's functions written in terms of the confluent hypergeometric functions.
The solution of the boundary value problem (8)-(9) was solved using the Runge-Kutta-Fehlberg integration scheme. In the method we choose finite values of $\eta \rightarrow \infty$. This value is the boundary layer thickness given by $\eta_{\infty}$. We begin by choosing an initial guess of $\eta_{\infty}$ to obtain the values $f^{\prime \prime}(0)$, $-\theta^{\prime}(0)$, and $-\phi^{\prime}(0)$. The solution is repeated with new values until two consecutive values differ by $10^{-6}$.

\section{Results and Discussion}

To obtain a clear understanding of the flow of Casson fluid, we discuss the physics of the problem by studying the effects of the unsteadiness $(A)$, permeability $(\Lambda)$, magnetic $(M)$, Prandtl (Pr), Schmidt (Sc), and reaction rate $(R)$ numbers on velocity, temperature, and concentration profiles. We also study the variation of skin friction, the local Nusselt number, and the Sherwood number with unsteadiness parameter. For validation of the numerical method used in this study, results for the Nusselt number $-\theta(0)$ for the Newtonian fluid were compared to those of Abd El-Aziz [3] and Grubka and Bobba [4] for the unsteadiness parameter $A=\Lambda=M=$ $\mathrm{Sc}=0$. The comparison is shown in Table 1 and it is found to be in agreement with at least four decimal places. To further verify the accuracy of the numerical scheme used, the successive linearization method (SLM) was used and there was agreement with Runge-Kutta-Fehlberg integration scheme.

To get a clear understanding of the behavior of velocity, temperature, and concentration profiles of Casson fluid, a detailed numerical calculation is done for different parameter values that describe the nature of flow and the results are depicted through Figures 1-5. 


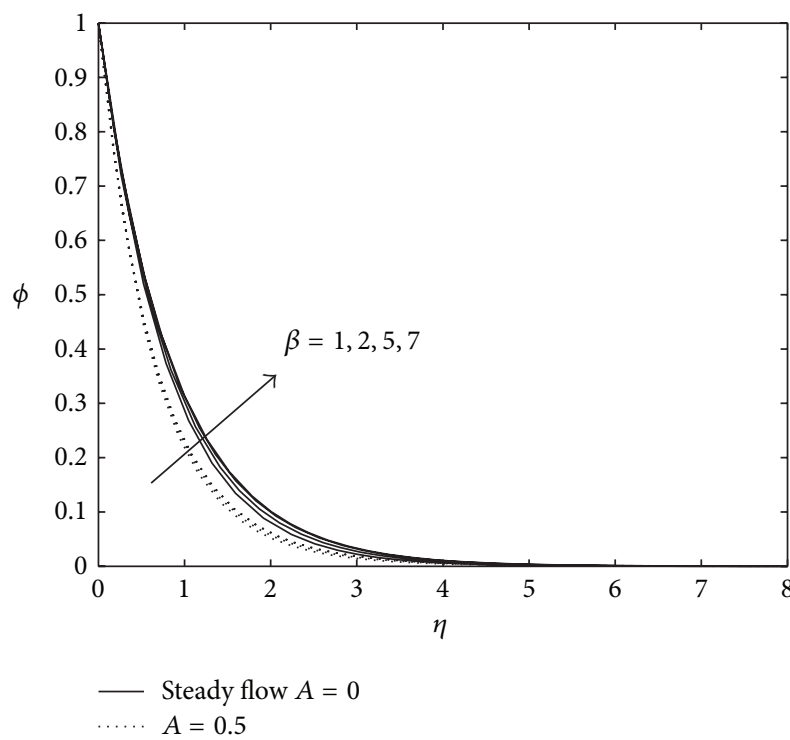

(a) Concentration profiles for different values of the Casson parameter $\beta$ and unsteadiness parameter $A$ at $\Lambda=0.5, M=1, \operatorname{Pr}=1, \mathrm{Sc}=1$, and $R=0.5$

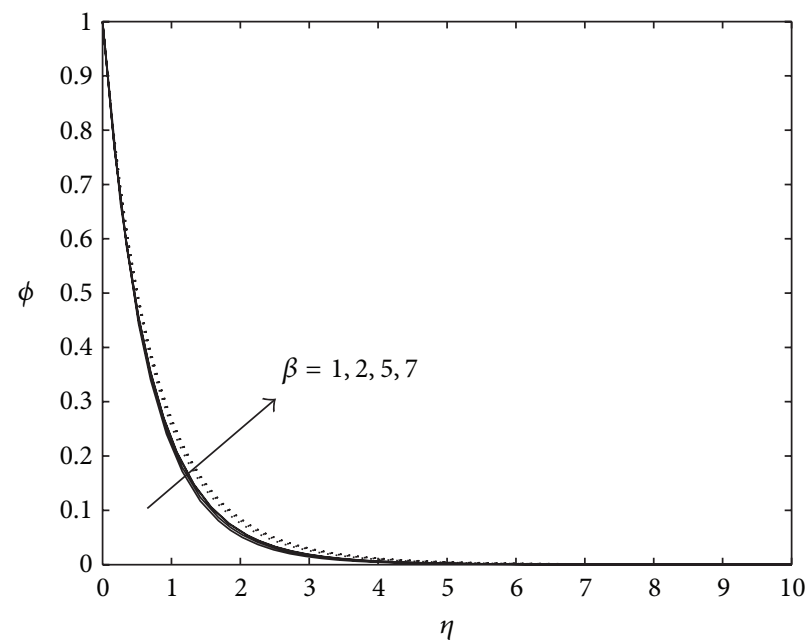

$$
M=1
$$$$
\text { …. } M=3
$$

(c) Concentration profiles for different values of the Casson parameter $\beta$ and magnetic parameter $M$ at $A=0.5, \Lambda=0.5, \operatorname{Pr}=1, \mathrm{Sc}=1$, and $R=0.5$

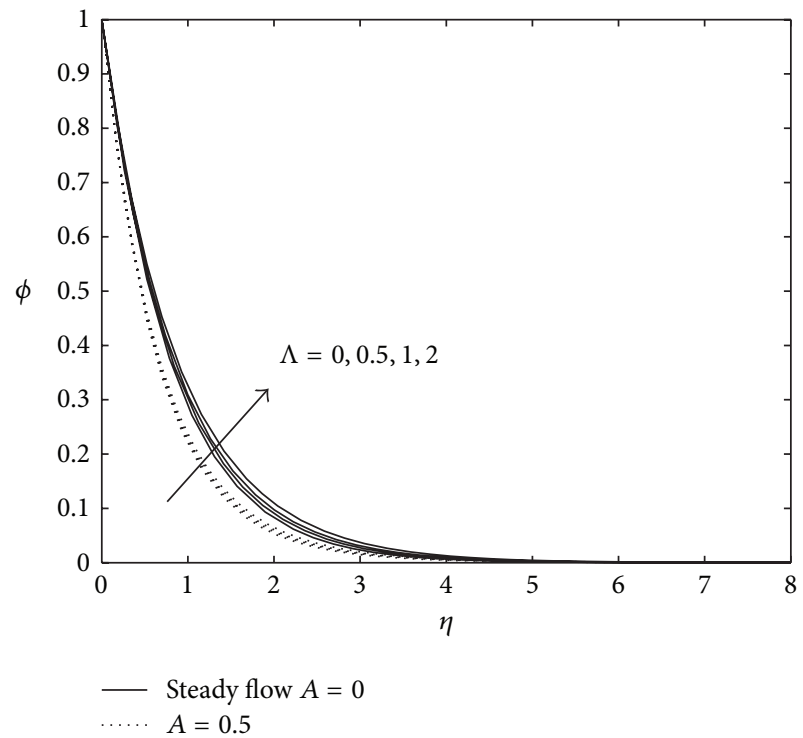

(b) Concentration profiles for different values of the permeability parameter $\Lambda$ and unsteadiness parameter $A$ at $\beta=2, M=1, \operatorname{Pr}=1$, $\mathrm{Sc}=1$, and $R=0.5$

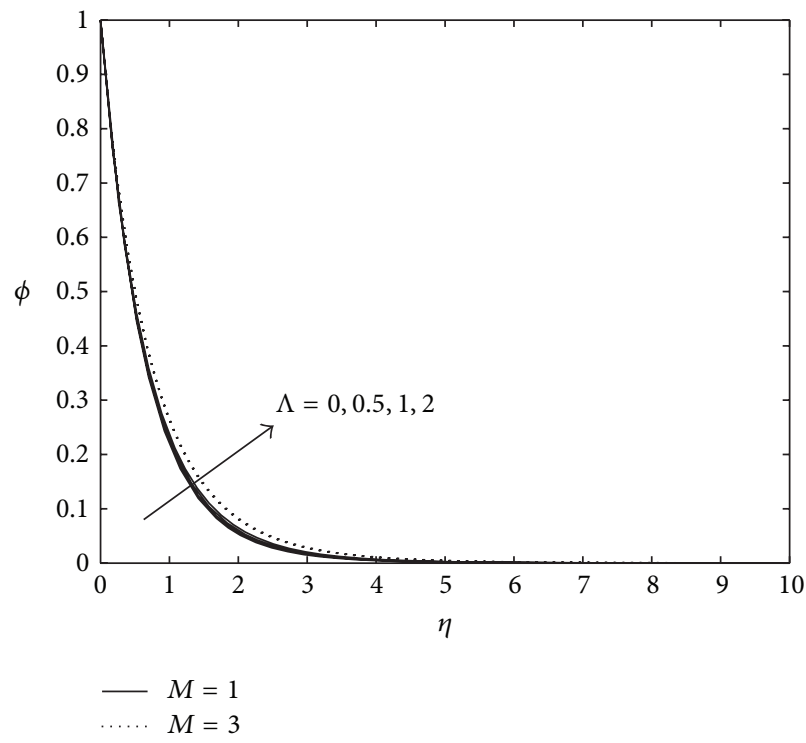

(d) Concentration profiles for different values of the permeability parameter $\Lambda$ and magnetic parameter $M$ at $A=0.5, \beta=2, \operatorname{Pr}=1$, $\mathrm{Sc}=1$, and $R=0.5$

FIGURE 4: Effects of different parameters on concentration profiles.

TABLE 1: Comparison of the values of $-\theta(0)$ for $\Lambda=M=\mathrm{Sc}=0$ and various values of $A$ and $\operatorname{Pr}$ with previously published data.

\begin{tabular}{cccccc}
\hline$A$ & Pr & Grubka and Bobba [4] & Abd El-Aziz [3] & SLM & Present results \\
\hline 0 & 0.72 & 0.8086 & 0.80873135 & 0.80873007 & 0.80863761 \\
& 1 & 1.0000 & 1.00000000 & 1.00000000 & 1.00000006 \\
& 1.9237 & 1.92368255 & 1.92367361 & 1.92367736 \\
& 3 & 3.7207 & 3.72067395 & 3.72066225 & 3.72066701 \\
\hline
\end{tabular}




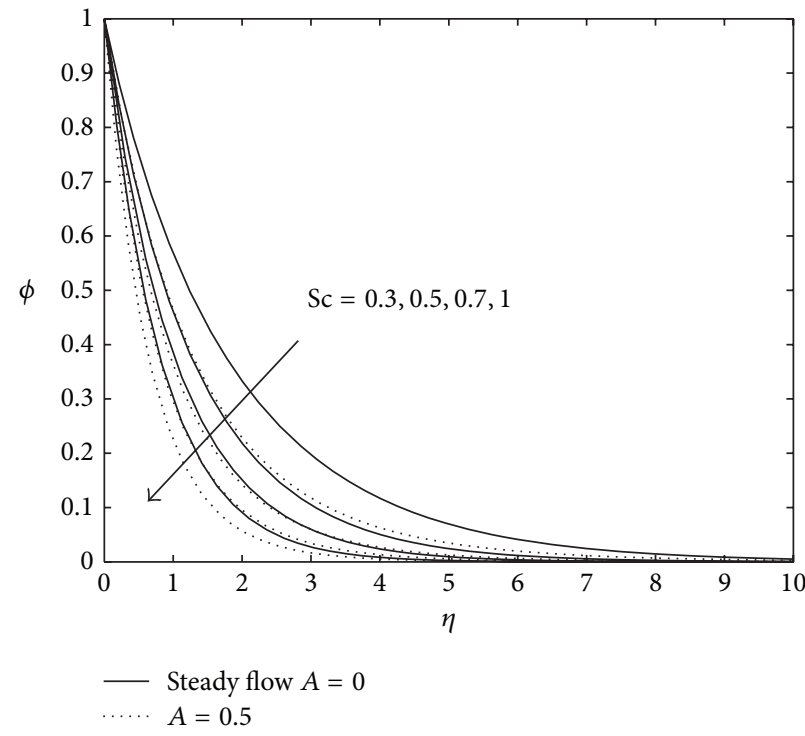

(a) Concentration profiles for different values of the Schmidt number Sc and unsteadiness parameter $A$ at $\beta=2, \Lambda=0.5, \operatorname{Pr}=1, M=1$, and $R=0.5$

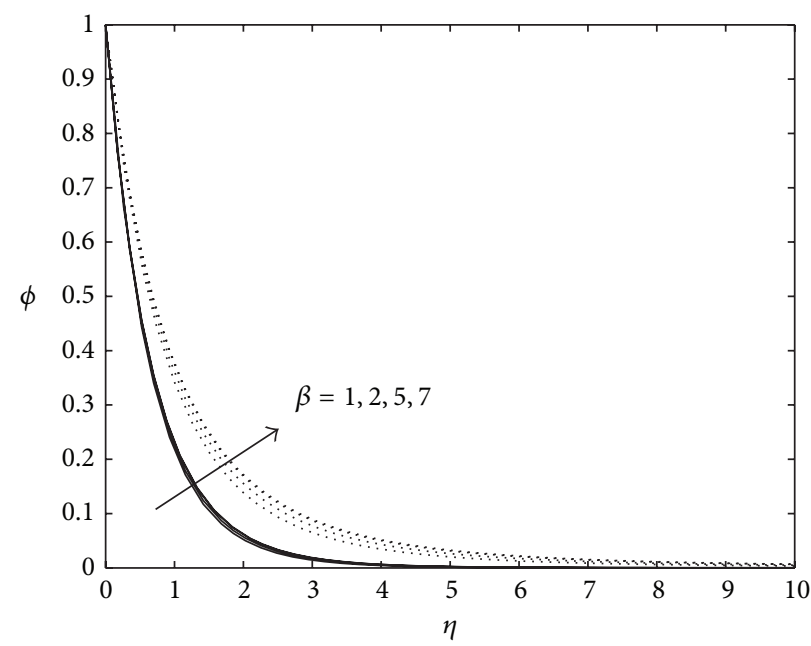

- Destructive reaction $R=0.5$

..... Constructive reaction $R=-0.5$

(c) Concentration profiles for different values of the Casson parameter $\beta$ and reaction rate $R$ at $A=0.5, M=1, \Lambda=0.5, \operatorname{Pr}=1$, and $\mathrm{Sc}=1$

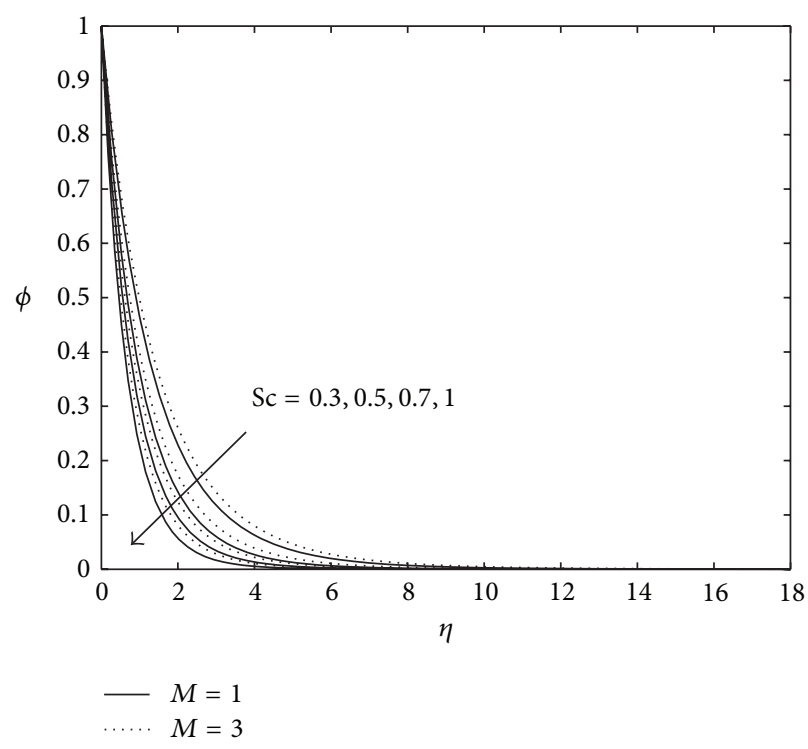

(b) Concentration profiles for different values of the Schmidt number Sc and magnetic parameter $M$ at $A=0.5, \beta=2, \Lambda=0.5, \operatorname{Pr}=1$, and $R=0.5$

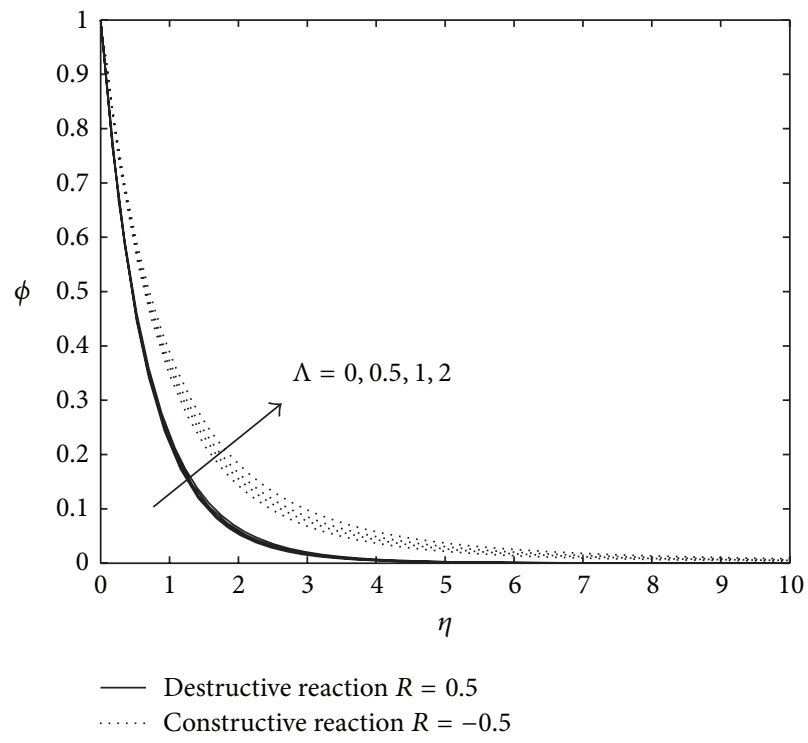

(d) Concentration profiles for different values of the permeability parameter $\Lambda$ and reaction rate $R$ at $A=0.5, \beta=2, M=1, \operatorname{Pr}=1$, and $\mathrm{Sc}=1$

FIGURE 5: Effects of different parameters on concentration profiles.

Figure 1 shows that increasing the Casson parameter $\beta$ and the permeability parameter $\Lambda$ decreases velocity profiles. Both situations retard fluid motion across the boundary layer as shown in Figures 1(a) to 1(d). The velocity profiles for unsteady flow become similar to steady flow far away from the boundary at $\eta>2$. This indicates that for $A=0.5$ the flow away from the boundary is steady. Increasing $A$ retards velocity profiles across the boundary layer as shown in Figures 1(a) and 1(b). In Figure 1(b) it is noted that increasing $A$ reduces velocity profiles and the reverse effect is noted around $\eta=2.5$ indicating slightly steady flow far away from the boundary. A slightly different observation is noted if the magnetic parameter is increased from $M=1$ to $M=3$; the velocity profiles decrease significantly reducing the momentum boundary layer thickness as shown in Figures $1(\mathrm{c})$ and $1(\mathrm{~d})$.

Figure 2 shows the variation of different parameters with temperature profiles. Increasing the Casson parameter increases temperature profiles; this decreases the yield stress. The fluid behaves as Newtonian as $\beta$ increases; this retards fluid motion. The effect of increasing $\beta$ leads to enhancing the temperature profiles for both steady and unsteady flows 


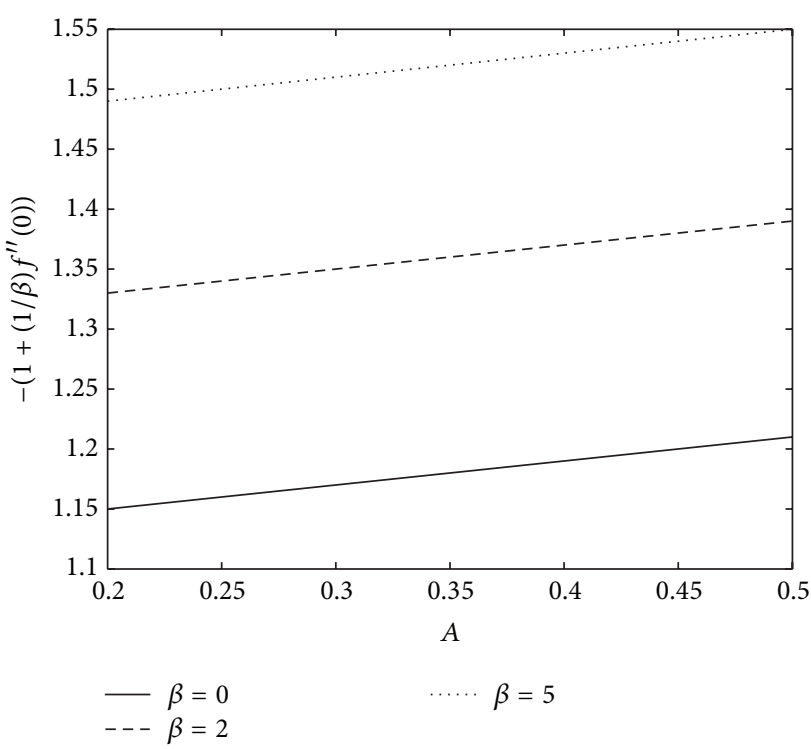

(a) Skin friction versus unsteadiness parameter at different values of the Casson parameter $\beta$ at $M=1, \Lambda=0.5, \operatorname{Pr}=1, \mathrm{Sc}=1$, and $R=0.5$

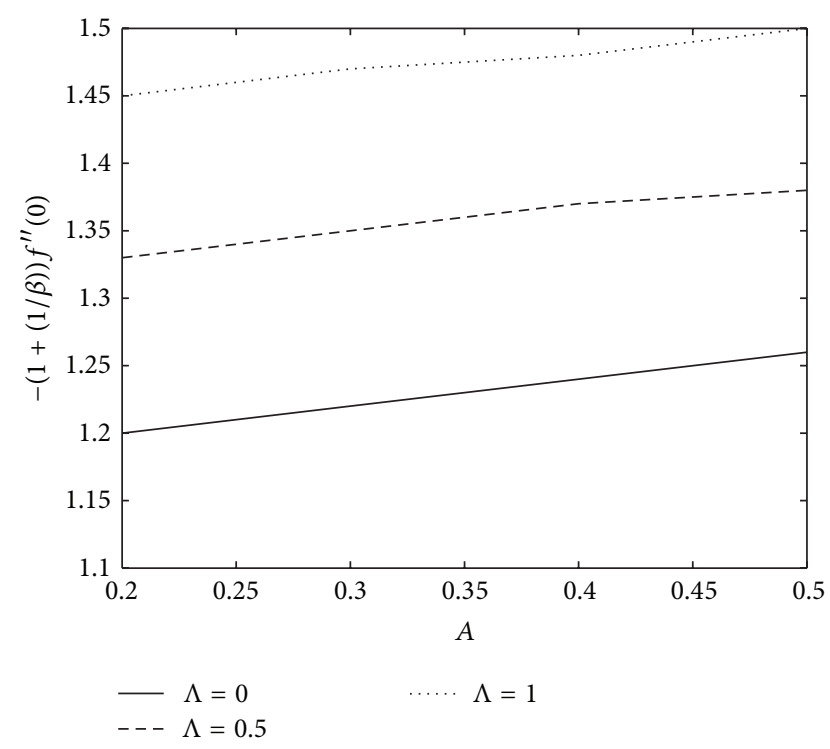

(b) Skin friction versus unsteadiness parameter at different values of the permeability parameter $\Lambda$ at $M=1, \beta=2, \operatorname{Pr}=1, \mathrm{Sc}=1$, and $R=0.5$

FIGURE 6: Variation of (a) skin friction coefficient with unsteadiness parameter for different $\beta$ values and (b) with different $\Lambda$ values.

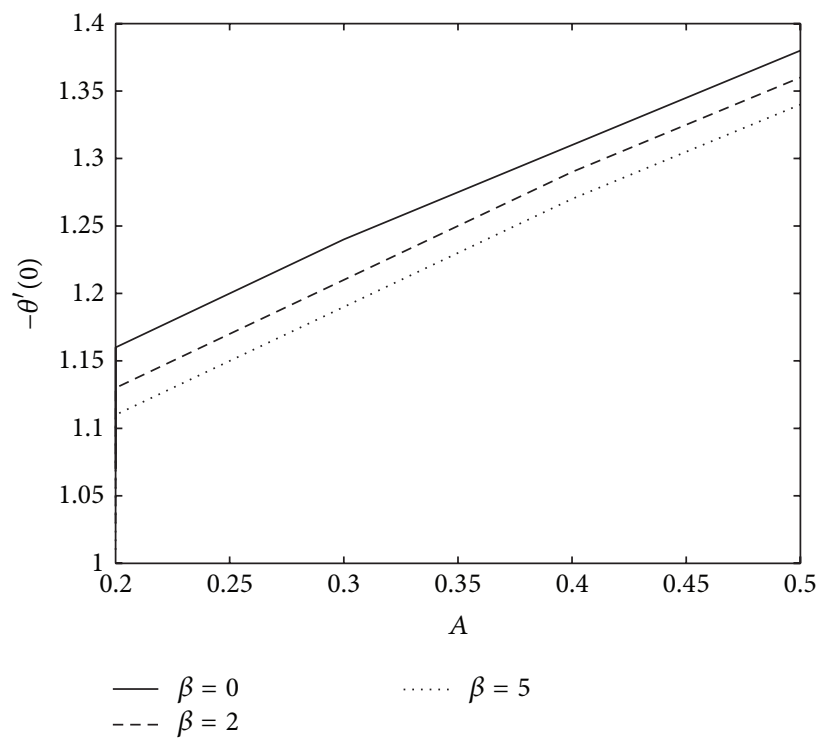

(a) Heat transfer coefficient versus unsteadiness parameter at different values of the Casson parameter $\beta$ at $M=1, \Lambda=0.5, \operatorname{Pr}=1, \mathrm{Sc}=1$, and $R=0.5$

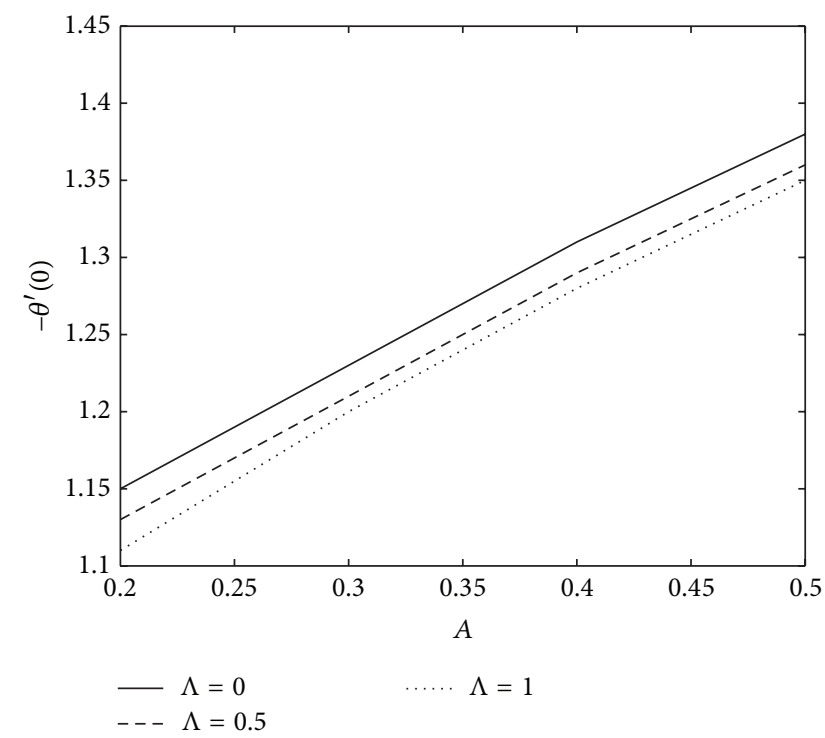

(b) Heat transfer coefficient versus unsteadiness parameter at different values of the permeability parameter $\Lambda$ at $M=1, \beta=0.5, \operatorname{Pr}=1, \mathrm{Sc}=1$, and $R=0.5$

FIGURE 7: Variation of (a) heat transfer coefficient with unsteadiness parameter for different $\beta$ values and (b) with different $\Lambda$ values.

as shown in Figure 2(a). The same observation is noted in Figure 2(d). Increasing the permeability parameter $\Lambda$ enhances temperature profiles. High values of $\Lambda$ lead to the reduction of fluid velocity enhancing temperature profiles. This effect is more pronounced in steady flow and the thickening of the thermal boundary layer increases as $\Lambda$ increases. In Figure 2(c) increasing the Prandtl number $\mathrm{Pr}$ reduces temperature profiles; $\mathrm{Pr}$ is inversely proportional to the thermal diffusivity which is low for Casson fluid. In Figures 2(d), 3(a), and 3(b), increasing $M$ increases temperature profiles; the slowing down of fluid flow leads to the buildup of heat. It is more pronounced in low values of $M$. Increasing $A$ leads to the reduction of temperature profiles; less heat is transferred from the sheet to the fluid; hence, temperature decreases as depicted in Figures 2(a), 2(b), and 2(c).

In Figure 4, increasing the Casson parameter $\beta$ results in the increase of the solute concentration; as the fluid 


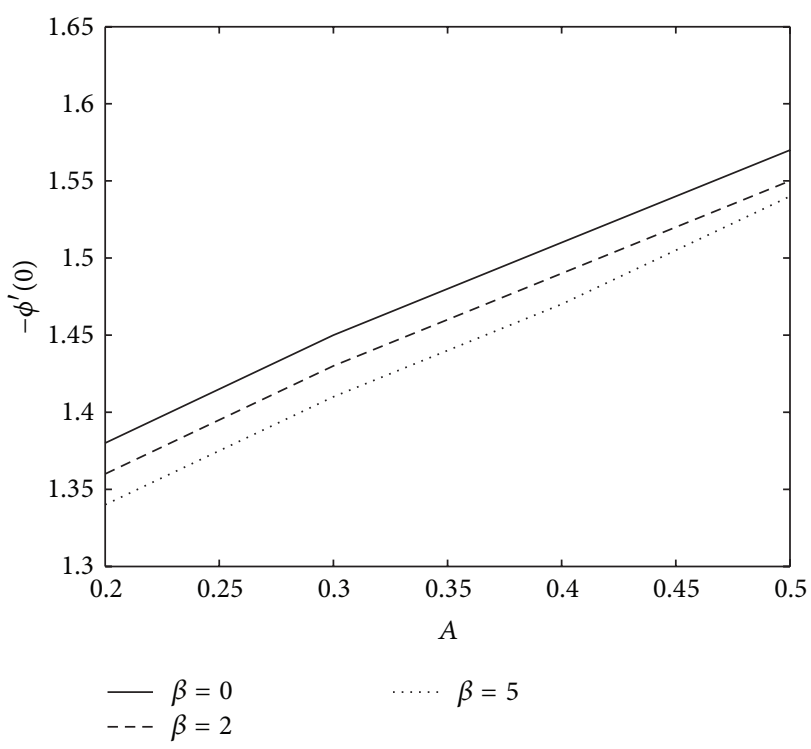

(a) Mass transfer coefficient versus unsteadiness parameter at different values of the Casson parameter $\beta$ at $M=1, \Lambda=0.5, \operatorname{Pr}=1, \mathrm{Sc}=1$, and $R=0.5$

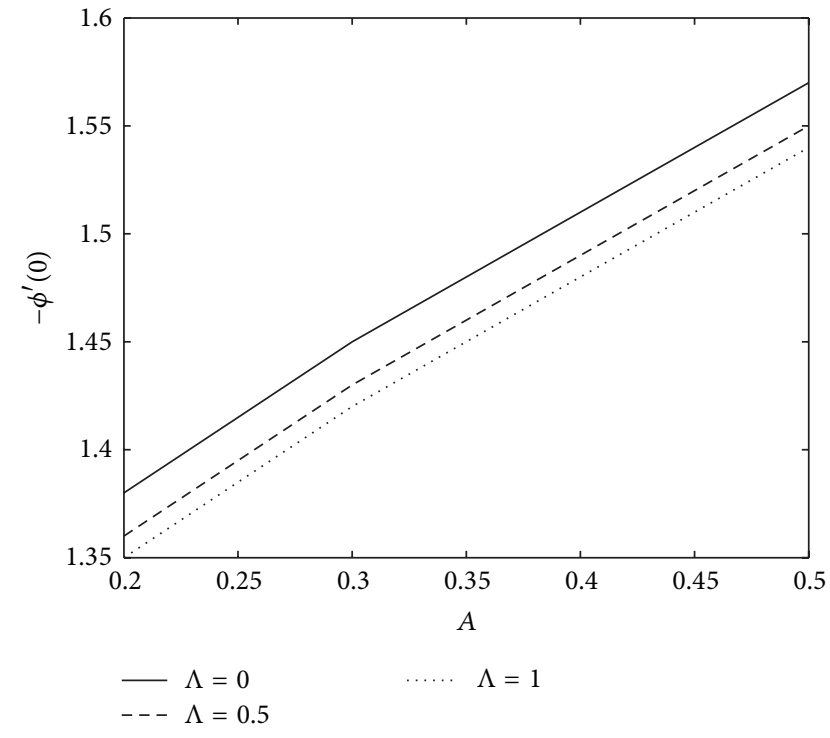

(b) Heat transfer coefficient versus unsteadiness parameter at different values of the permeability parameter $\Lambda$ at $M=1, \beta=2, \operatorname{Pr}=1, \mathrm{Sc}=1$, and $R=0.5$

FIGURE 8: Variation of (a) mass transfer coefficient with unsteadiness parameter for different $\beta$ values and (b) with different $\Lambda$ values.

becomes Newtonian, solute transfer is enhanced as shown in Figures 4(a), 4(d), and 5(c). This is more pronounced in steady flow. The opposite effect is noted in increasing $A$ which reduces solute concentration. Increasing $\Lambda$ increases concentration profiles; since this reduces fluid motion, it leads to the buildup of solute concentration as shown in Figures 4(b), 4(d), and 5(d). Increasing Sc decreases concentration profiles; the diffusion rate is smaller in Casson fluid and hence reducing solute concentration as depicted in Figures 5(a) and 5(b). In Figures 4(c), 4(d), and 5(b), increasing $M$ increases profiles; the slowing down of fluid flow leads to the buildup of mass. It is more pronounced in low values of $M$.

Figures 5(c) and 5(d) show the effects of reaction rate $R$ on the concentration profiles in Casson fluid in porous medium. Increasing the absolute value of $R$ decreases the concentration profiles. The reaction rate is in general a destructive agent which leads to the reduction of the solute boundary layer. It shows the increase of solute boundary layer in the case of constructive reaction $R<0$ and the decrease of solute boundary layer in the case of destructive reaction $R>0$. The effect of increasing $\beta$ and $\Lambda$ is more pronounced in unsteady flow.

Figures 6(a) and 6(b) show effects of Casson parameter $\beta$ and permeability parameter $\Lambda$ on velocity gradient at the wall with unsteadiness parameter. The magnitude of $f^{\prime \prime}(0)$ related to skin friction decreases with increasing unsteadiness parameter $A$ and also with Casson parameter $\beta$ and permeability parameter $\Lambda$. The magnitude of the heat transfer rate at the surface $-\theta^{\prime}(0)$ decreases with increasing $\beta$ and $\Lambda$ and increases with $A$ as shown in Figure 7. The mass transfer coefficient at the surface $-\phi^{\prime}(0)$ decreases with increasing $\beta$ and $\Lambda$ and increases with $A$ as shown in Figure 8.

\section{Conclusion}

The study presented in this analysis of diffusion of chemically reactive species in Casson fluid flow over an unsteady stretching surface in a porous medium in the presence of a magnetic field provides numerical solutions for the boundary layer flow and heat and mass transfer. The coupled nonlinear governing equations were solved using the Runge-KuttaFehlberg integration scheme. Increasing the unsteadiness parameter decreases velocity profiles. Increasing the Casson parameter decreases the velocity profiles. The presence of heat transfer does not seem to significantly change the results obtained by Mukhopadhyay and Vajravelu [1]; the main difference is noted in the presence of magnetic and permeability parameters which they did not consider. In this study it is generally noted that increasing the magnetic parameter and permeability parameter decreases the velocity profiles but increases the skin friction; it then decreases the coefficient of heat transfer and the concentration profiles.

\section{Conflict of Interests}

The authors declare that there is no conflict of interests regarding the publication of this paper.

\section{References}

[1] S. Mukhopadhyay and K. Vajravelu, "Diffusion of chemically reactive species in Casson fluid flow over an unsteady permeable stretching surface," Journal of Hydrodynamics, vol. 25, no. 4, pp. 591-598, 2013. 
[2] S. Mukhopadhyay, P. R. de, K. Bhattacharyya, and G. C. Layek, "Casson fluid flow over an unsteady stretching surface," Ain Shams Engineering Journal, vol. 4, no. 4, pp. 933-938, 2013.

[3] M. Abd El-Aziz, "Mixed convection flow of a micropolar fluid from an unsteady stretching surface with viscous dissipation," Journal of the Egyptian Mathematical Society, vol. 21, no. 3, pp. 385-394, 2013.

[4] L. J. Grubka and K. M. Bobba, "Heat transfer characteristics of a continuous stretching surface with variable temperature," Journal of Heat Transfer, vol. 107, no. 1, pp. 248-250, 1985.

[5] S. Sharidan, T. Mahmood, and I. Pop, "Similarity solutions for the unsteady boundary layer flow and heat transfer due to a stretching sheet," International journal of Applied Mechanical Engineering, vol. 11, pp. 647-654, 2006.

[6] S. Nadeem, R. Ul Haq, and C. Lee, "MHD flow of a Casson fluid over an exponentially shrinking sheet," Scientia Iranica, vol. 19, no. 6, pp. 1550-1553, 2012.

[7] S. Nadeem, R. U. Haq, and Z. H. Khan, "Numerical study of MHD boundary layer flow of a Maxwell fluid past a stretching sheet in the presence of nanoparticles," Journal of the Taiwan Institute of Chemical Engineers, vol. 45, no. 1, pp. 121-126, 2014.

[8] K. Ahmed and R. Nazar, "Magnetohydrodynamic three dimensional flow and heat transfer over a stretching surface in a viscoelastic fluid," Journal of Science and Technology, vol. 3, no. 1, pp. 33-46, 2011.

[9] K. Rohlf and G. Tenti, "The role of the Womersley number in pulsatile blood flow a theoretical study of the Casson model," Journal of Biomechanics, vol. 34, no. 1, pp. 141-148, 2001.

[10] D. S. Sankar and U. Lee, "Two-fluid non-linear model for flow in catheterized blood vessels," International Journal of Non-Linear Mechanics, vol. 43, no. 7, pp. 622-631, 2008.

[11] D. S. Sankar and U. Lee, "Two-fluid Casson model for pulsatile blood flow through stenosed arteries: a theoretical model," Communications in Nonlinear Science and Numerical Simulation, vol. 15, no. 8, pp. 2086-2097, 2010.

[12] S. Shaw, R. S. R. Gorla, P. V. S. N. Murthy, and C.-O. Ng, "Pulsatile casson fluid flow through a stenosed bifurcated artery," International Journal of Fluid Mechanics Research, vol. 36, no. 1, pp. 43-63, 2009.

[13] A. V. Mernone, J. N. Mazumdar, and S. K. Lucas, "A mathematical study of peristaltic transport of a Casson fluid," Mathematical and Computer Modelling, vol. 35, no. 7-8, pp. 895912, 2002.

[14] B. Das and R. L. Batra, "Secondary flow of a Casson fluid in a slightly curved tube," International Journal of Non-Linear Mechanics, vol. 28, no. 5, pp. 567-577, 1993.

[15] C.-O. Ng, "Combined pressure-driven and electroosmotic flow of Casson fluid through a slit microchannel," Journal of NonNewtonian Fluid Mechanics, vol. 198, pp. 1-9, 2013.

[16] S. Nadeem, R. Ul Haq, N. S. Akbar, and Z. H. Khan, "MHD three-dimensional Casson fluid flow past a porous linearly stretching sheet," Alexandria Engineering Journal, vol. 52, no. 4, pp. 577-582, 2013.

[17] R. K. Dash, K. N. Mehta, and G. Jayaraman, "Casson fluid flow in a pipe filled with a homogeneous porous medium," International Journal of Engineering Science, vol. 34, no. 10, pp. 1145-1156, 1996.

[18] D. Tripathi, "Study of transient peristaltic heat flow through a finite porous channel," Mathematical and Computer Modelling, vol. 57, no. 5-6, pp. 1270-1283, 2013.
[19] S. Pramanik, "Casson fluid flow and heat transfer past an exponentially porous stretching surface in presence of thermal radiation," Ain Shams Engineering Journal, vol. 5, no. 1, pp. 205212, 2014.

[20] P. V. Ramachandra, R. A. Subba, and B. O. Anwa, "Flow and heat transfer of casson fluid from a horizontal cylinder with partial slip in non-darcy porous medium," Applied and computational Mathematics, vol. 2, article 2, 2013.

[21] P. K. Kameswaran, S. Shaw, P. Sibanda, and P. V. S. N. Murthy, "Homogeneous-heterogeneous reactions in a nanofluid flow due to a porous stretching sheet," International Journal of Heat and Mass Transfer, vol. 57, no. 2, pp. 465-472, 2013.

[22] S. Shaw, P. K. Kameswaran, and P. Sibanda, "Homogeneousheterogeneous reactions in micropolar fluid flow from a permeable stretching or shrinking sheet in a porous medium," Boundary Value Problems, vol. 2013, article 77, 2013.

[23] A. J. Chamkha, A. M. Aly, and M. A. Mansour, "Similarity solution for unsteady heat and mass transfer from a stretching surface embedded in a porous medium with suction/injection and chemical reaction effects," Chemical Engineering Communications, vol. 197, no. 6, pp. 846-858, 2010.

[24] G. Makanda, O. D. Makinde, and P. Sibanda, "Natural convection of viscoelastic fluid from a cone embedded in a porous medium with viscous dissipation," Mathematical Problems in Engineering, vol. 2013, Article ID 934712, 11 pages, 2013. 


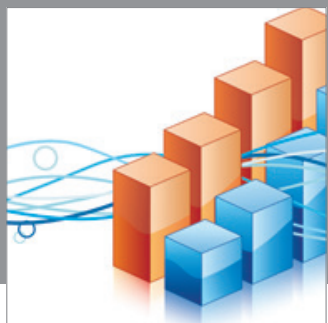

Advances in

Operations Research

mansans

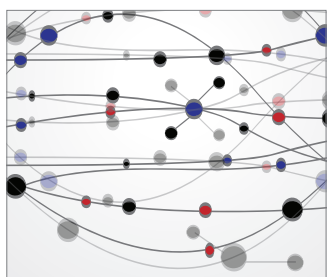

The Scientific World Journal
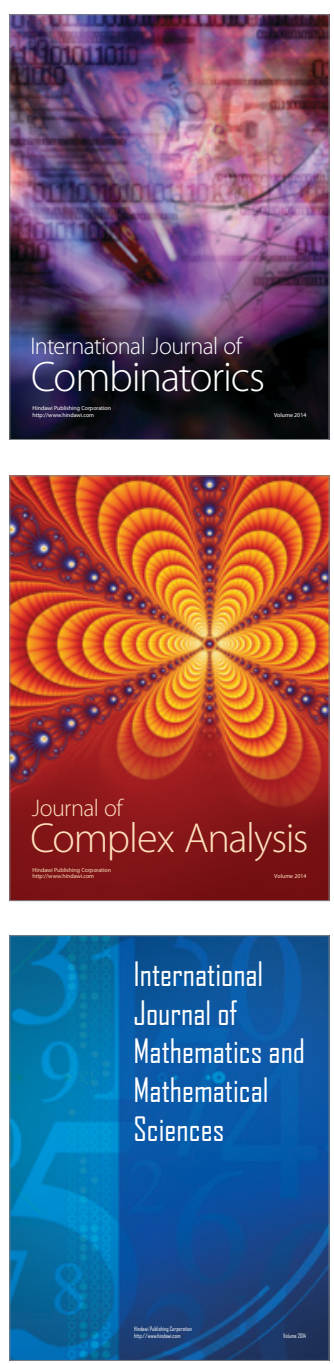
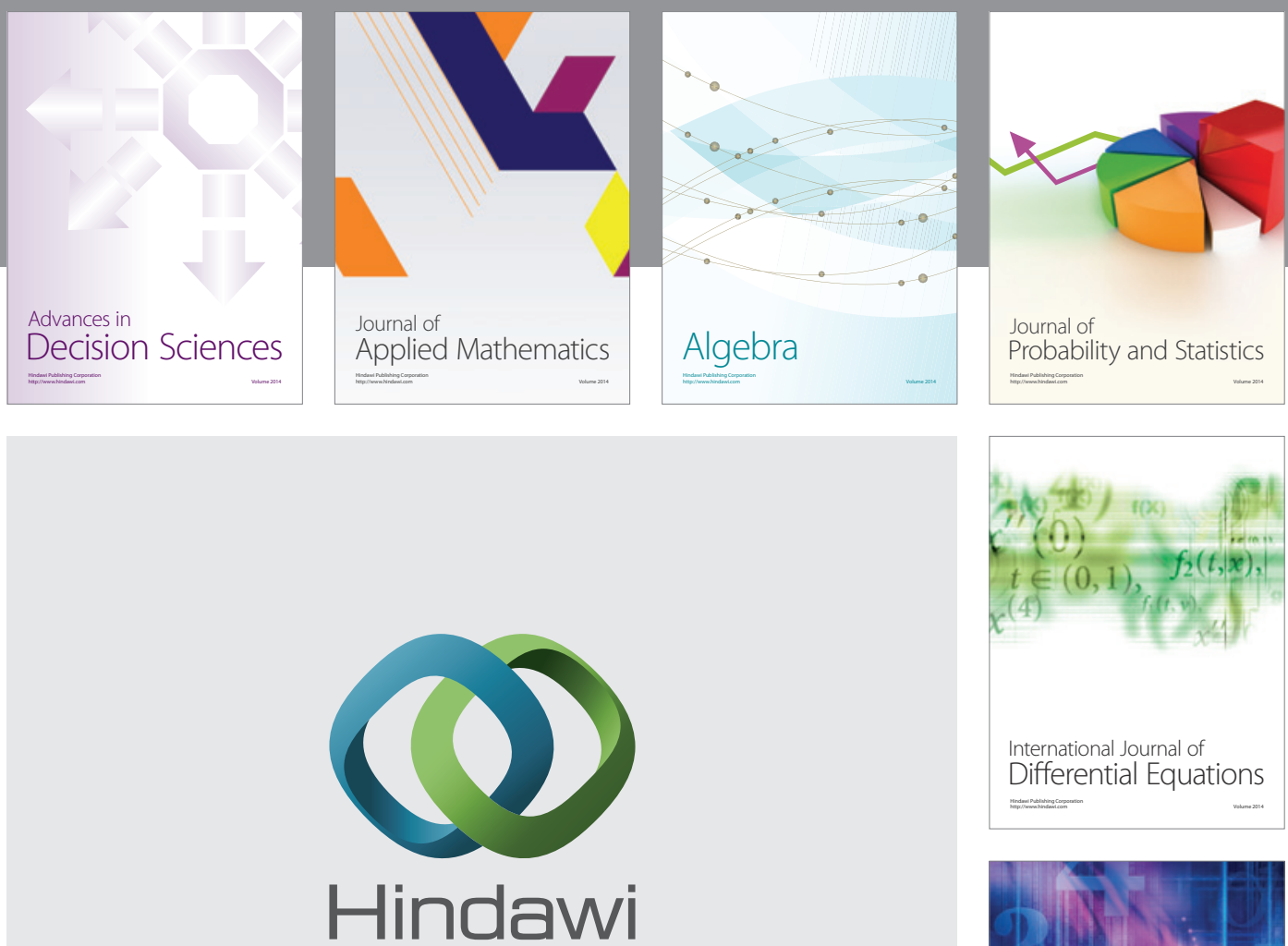

Submit your manuscripts at http://www.hindawi.com
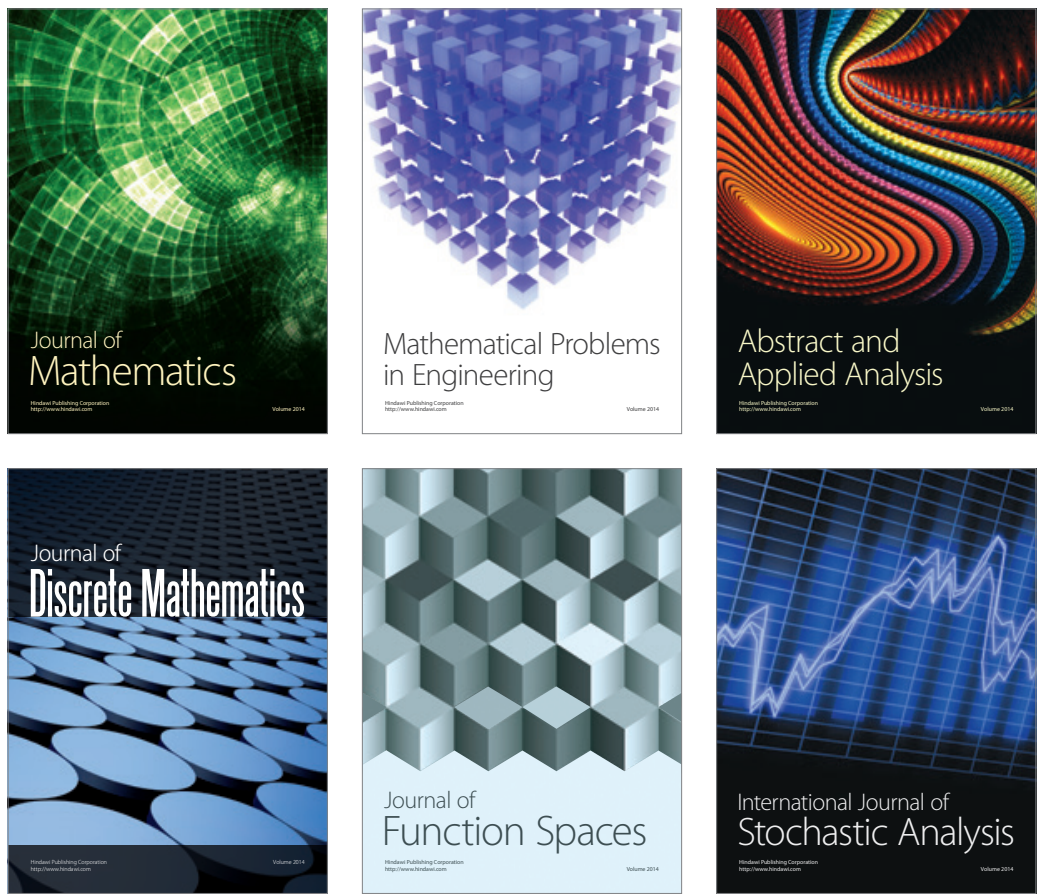

Journal of

Function Spaces

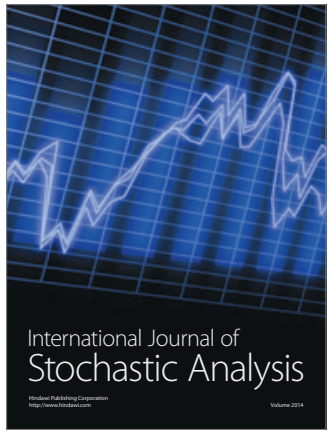

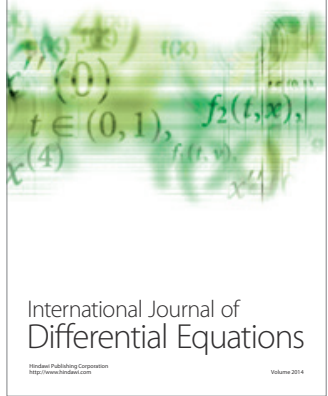
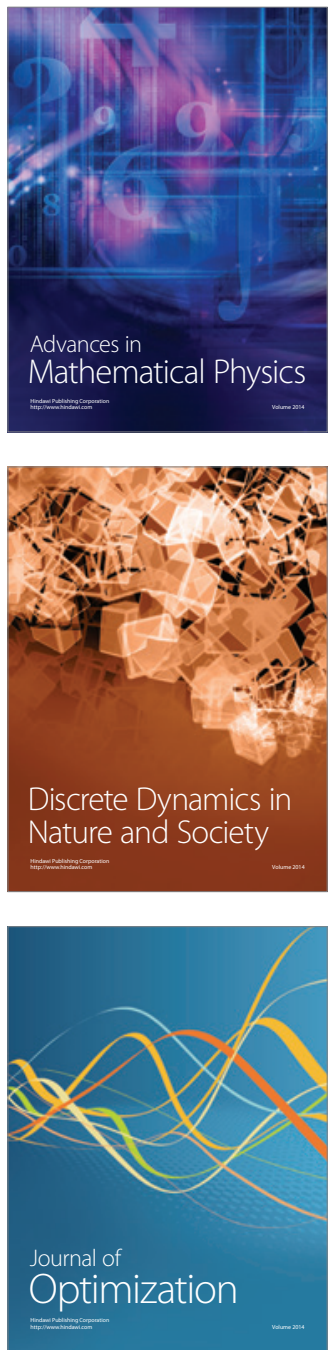